\title{
The Prevalence Rate of Cigarette Smoking among Congestive Heart Failure Patients at a Local Family Health Center
}

\author{
Salome Kapella-Mshigeni ${ }^{1 *}$, Eunice Kimunai ${ }^{2}$ and Chad L Cross ${ }^{3}$ \\ ${ }^{1}$ Department of Health Science and Human Ecology, College of Natural Science, California State University San Bernardino, \\ USA \\ ${ }^{2}$ CliniHealth Consultants Inc, USA \\ ${ }^{3}$ School of Community Health Sciences, University of Nevada, USA
}

\begin{abstract}
Introduction: The prevalence rate of smoking is well documented in the general population, but little is known among patients with Congestive Heart Failure (CHF). The mortality rate of CHF in San Bernardino county is nearly double (18.7 per 100,000 deaths) than that of the State of California (10.8 per 100,000 deaths). There is a need to fill knowledge gaps for $\mathrm{CHF}$ patients and identify evidence-based interventions that will help reduce health disparities among populations.

Objective: This study aimed to determine the prevalence rate of cigarette smoking among an underserved population for patients with CHF seen at a Family Health Clinic in San Bernardino County. It also sought to gain a quantitative understanding of smoking prevalence rates among past and current smokers with the goal of providing evidence-based interventions for healthcare professionals.

Methods: This study used secondary data for CHF patients who self-reported their existing social history as documented by their provider. The list was accessed using Patient Registry report functionality and a further search was conducted using their Electronic Medical Record (EMR).

Results: $58 \%$ reported smoking in the past and $27 \%$ reported to be current smokers. Hence, among CHF patients, the prevalence rate of current smokers is significantly less than past smokers.

Conclusions: These findings demonstrate the effectiveness of using evidence-based practice at a county clinic that serves the underserved population. Patients with chronic diseases (i.e. heart failure) are empowered to take an active role in their health to reduce the number of Emergency Room visits. The results from this study will aid public health professionals, healthcare providers, and supporting agencies in creating evidence-based interventions for smokers with $\mathrm{CHF}$ with the goal of reducing health disparities and alternatively decrease their overall disease burden. Further research opportunities that will utilize secondary data from multiple study sites are highly recommended.
\end{abstract}

\section{Keywords}

Cigarette, Smoking, Congestive heart failure, Interventions

\section{Introduction}

The prevalence rate of smoking is well documented in the general population; however, little is known about cigarette smoking among patients with Congestive Heart Failure (CHF). According to the Centers for Disease Control and Prevention (CDC), heart failure occurs when a heart becomes weak and is not able to pump blood throughout the body [1]. There are 5.7 million people in the United States with CHF, causing 1 in 9 deaths in 2009 [2]. Research also shows that about half of the patients who are diagnosed with CHF die within 5 years of their diagnosis [3]. The mortality rate of $\mathrm{CHF}$ in California alone was 10.8 per 100,000 deaths while that of San Bernardino County was almost double the state's statistic at 18.7 - 31.6 per 100,000 [4]. Financially, the United States spends an average of $\$ 32$ billion per year on the provision of treatment services, disease management, and missed productivity time from work for patients with CHF [3]. Every

*Corresponding author: Salome Kapella-Mshigeni, PhD, MPH, MPA, Department of Health Science and Human Ecology, College of Natural Science, California State University San Bernardino, 5500 University Parkway, San Bernardino, CA 92407, USA

Accepted: January 17, 2019

Published online: January 19, 2019

Citation: Kapella-Mshigeni S, Kimunai E, Cross CL (2019) The Prevalence Rate of Cigarette Smoking among Congestive Heart Failure Patients at a Local Family Health Center. Ann Public Health Reports 3(1):30-34 
Citation: Kapella-Mshigeni S, Kimunai E, Cross CL (2019) The Prevalence Rate of Cigarette Smoking among Congestive Heart Failure Patients at a Local Family Health Center. Ann Public Health Reports 3(1):30-34

effort into factors affecting CHF management is therefore crucial to the overall population health agenda.

As of 2015, the prevalence rate of cigarette smoking in the United States was 15.1 percent, with 37.8 million adults reported to be current cigarette smokers and more than 16 million of them living with a smoking related disease [5]. Research indicates that 7 out of 10 smokers have expressed a desire to quit smoking but lack the necessary resources or professional support to follow through. One out of every 5 deaths in the United States has been attributed to cigarette smoking despite the fact that it is one of the most preventable causes of deaths and illnesses in the U.S. $[5,6]$.

In California, the prevalence rate of cigarette smoking among adults was 11.7 percent [7]. Specifically, in San Bernardino County, the prevalence rate of cigarette smoking among adults was higher than the state average, at 14.6 percent [7]. There is no current data pertaining to the prevalence rate of cigarette smoking among CHF patients in California.

This study was therefore designed to investigate the prevalence rate of cigarette smoking among $\mathrm{CHF}$ patients from a primary care setting in San Bernardino county with the goal of proposing evidence-based interventions that best fit this population. Healthcare practitioners as well as patients with CHF from the McKee Family Health Center (FHC), one of the Ambulatory Clinics at Arrowhead Regional Medical Center (ARMC), will benefit from the following: an increased awareness of the prevalence rate of cigarette smoking among CHF patients and recommendations for future interventions on evidence-based health management.

\section{Literature review}

Tobacco use, specifically cigarette smoking, continues to be the leading cause of preventable morbidity and mortality in the United States, with a third of its users addicted to the drug nicotine $[2,8,9]$. Cigarette smoking results in more than 480,000 premature deaths in the U.S. annually, accounting 1 out of every 5 deaths because of smoking $[5,8]$. According to the NIH, smoking is a key risk factor for heart disease, and when combined with other unhealthy conditions such as elevated cholesterol level, high blood pressure, obesity, and smoking intensifies the risk of heart disease including: heart attack, heart failure, or chest pain $[2,6]$.

Some of the well-documented risk factors for $\mathrm{CHF}$ include: Coronary Heart Disease (CHD), Hypertension (HTN), and Diabetes [2]. Additionally, unhealthy behavioral patterns also contribute to the risk of CHF development specifically for people with CHD, HTN, and Diabetes $[2,6]$. Other behavioral risk factors include: Consumption of foods that are high in saturated fats, high cholesterol, high sodium intake, and lack of physical activity/obesity [2].

One goal of Healthy People 2020 (HP2020) is to reduce hospitalizations of older adults with heart failure as the principal diagnosis [10]. Currently, CHF has no cure $[2,3,10,11]$. However, treatments such as pharmacological interventions and lifestyle changes can help people who have the condition live longer [3]. Promisingly, researchers continue to study new ways to treat $\mathrm{CHF}$ and its accompanying complications. Research indicates that children and adults are impacted by this disease, but that symptoms and treatments for each age group do differ [3].

As it was once suggested by the Former U.S. Surgeon General Director David Satcher, MD, PhD, tobacco continues to be the single greatest preventable cause of disease and premature death in America and hence every doctor, nurse, health plan, purchaser, and medical school in America should make treating tobacco dependence a top priority [12]. Healthcare providers in primary care settings have a vital role in encouraging their patients to quit smoking, particularly those suffering with chronic conditions such as $\mathrm{CHF}$, cardiovascular diseases, respiratory diseases, diabetes, several types of cancer, and stroke [13]. As research has shown, empowering patients with information that increases their health knowledge and collaborating with them in the creation of self-management goals will help make changes in their health behaviors and is the best approach to chronic disease management [14].

Evidence-based smoking cessation methods have been widely promoted and have succeeded in reducing tobacco use in the United States. According to the CDC, the utilization of evidence-based statewide tobacco control programs that are comprehensive, sustained, and accountable tend to reduce the prevalence rate of smoking and subsequently the number of tobacco-related diseases and deaths [15]. Research supports that offering smoking cessation interventions to patients with chronic conditions during hospital stay is effective as long as the interventions are continued one month after discharge from the hospital $[16,17]$. The combined intervention of behavioral support (i.e. counseling and brief advice) and medications use (i.e. nicotine replacement therapies, varenicline, and bupropion) has been documented as the most effective approach instead of one intervention alone [16-18]. Stead, et al. [18] found that a combined intervention increases the chances of successfully quitting by 70 to 100 percent after at least six months compared to just behavioral support. Success for quitting tended to be larger when participants were recruited in hospital settings [18].

Assistance with smoking cessation is therefore a fundamental element of the management of the patients with chronic conditions, such as CHF. Health teams have a professional obligation to assist with the initiation of smoking cessation interventions and advance tobacco control efforts. Broader dissemination, adoption, and implementation of evidence-based interventions are keys to addressing the tobacco epidemic [19].

\section{Research objectives}

This study aimed to determine the prevalence rate of cigarette smoking among patients with $\mathrm{CHF}$ seen at one local Family Health Clinic in San Bernardino County, California. It also sought to gain a quantitative understanding of smoking prevalence rates among past and current smokers in order to identify other areas of investigation that will aid in the formulation of more effective population health strategies. 


\section{Materials and Methods}

This study utilized secondary data that was previously collected for healthcare service purposes, whereby patients' records were retrieved by the Health Educator from the clinic patient registry. 198 out of the 241 patient records collected were selected as suitable for analysis based on the following inclusion criteria: Adults ages 21 to 75 with a new or current diagnosis of CHF who have been seen in the clinic within the study period (January 1, 2015 - December 31, 2015).

Patients were excluded if they were above 75 years of age, owing to the possibility of dementia and disability complicating their response accuracy. All ethical guidelines were followed, and a safety protocol secured data retrieval throughout the study. Only the Principal Investigator, CoInvestigators, and Research Coordinator had access to patient information used in the study. The Research Coordinator removed all patient identifiers from the selected sample prior to data analysis.

Prevalence rates for past and current smokers were calculated as a simple ratio of those answering "yes" or "no" to smoking to the total number of observations. To test for significant differences between prevalence rates of past and current smokers, an exact test of proportions with continuity correction was employed. All quantitative data analyses were completed in R (v. 3.3.1; https://cran.r-project.org/).

\section{Interventions used}

The nurses; doctors, and medical assistants utilized an evidence-based method to assess for cigarette smoking status known as the 3As: Ask; Advise and Refer patients to a quit clinic resource as recommended by Agency for Healthcare Research and Quality [12]. This smoking cessation approach has been proven to work for both parties: Healthcare professionals as well as patients as it can be implemented in less than 90 seconds. Quit clinic resources include: Over-the-phone or face-to-face counseling sessions. Healthcare professionals from the McKee FHC utilized it to check for smoking status among their CHF patients and later on referred them to a Quit clinic inside the Hospital or over the phone counseling session i.e. the California Smokers Helpline.

\section{Theory}

The stages of change theory, (also known as the transtheoretical model of behavioral change) as put forth by Prochaska and Goldstein [20] is the best fit framework for this study as it has been proven to work well in many smoking cessation efforts. This theory is utilized in many public health strategies, such as when patients try to lose weight, manage their chronic diseases, eat healthy, quit smoking, or stop any other possible addictions that affects physical, mental, and emotional well-being [21]. It provides a patient-centered counseling intervention approach for smoking cessation as well as many other related health behaviors. This theory involves 5 stages of change that individuals often go through prior to making a committment to accomplishing their goals in many public health related prevention activies.

The stages are: Precontemplation, contemplation, preparation, action, and maintanance [20]. When it comes to smoking cessation among heart failure patient, just like any other intervention, patients go through precontemplation, wondering if they even have a problem with their daily smoking. During the contemplation stage, individuals begin to think about change, but they have yet to allow their inner self to let that mental committment take place. At the preparation stage, this is when individuals are ready to change and make a committment such as the decision to quit smoking. During the action stage, individuls are making an actual change, and this is when they actually make efforts to quit i.e. "set a quit date within the next 30 days, and begin counseling sessions from a professional cessation program". Maintenance stage is the last and fifth part where individuals stay on track and work very hard to maintain the actual change (e.g., abstain from smoking by exercising, eating healthy, and feeling good about their accomplishment).

This theory supports a cycle of change with many possibilities of relapse before individuals achieve their goals (e.g., to quit smoking; [20]). It is useful in identifying positive behavioral transformation whereby an individual is in the change process [22]. When applied to smoking cessation programs, this theory allows programs to monitor interventions and provide constructive feedback that will prevent the subjects from rushing through the process or moving too slowly. The use of this theory often helps programs complete stage-matched, patient-centered counseling interventions that often help physicians feel less frustrated in the provision of care $[20,23]$.

\section{Results}

Findings showed an alarming prevalence rate $(27 \%)$ of smoking among adult CHF patients seen at the McKee FHC compared to the general population's prevalence rates (16.8\%) for the country [5] and (11.7\%) for the State of California [7]. Knowing that cigarette smoking has continually been shown as a key risk factor for heart disease morbidity and mortality, this study presented an even more urgent need to formulate effective evidence-based interventions for not only the McKee FHC patients but also other CHF patients in the country.

In the context of evidence-based strategies for health management, the statistical difference between $\mathrm{CHF}$ patients who were past smokers and current smokers' points to additional questions that will help tailor more effective interventions for the clinic's constituents. For example, of the past smokers who are not current smokers, "what made them quit?" will provide a wealth of information about population-specific motivation factors that could be used in client education projects. At the same time, differences between the past smokers' and current smokers' access to health resources can further be investigated to identify gaps in population health services. Certainly, the use of the $3 \mathrm{~A}$ approach: Ask, Advise, and Refer to a cessation program has helped reduce the prevalence rate, however more needs to be done to lower the numbers much further.

\section{Study implications}

Findings from this study will aid public health profession- 
Citation: Kapella-Mshigeni S, Kimunai E, Cross CL (2019) The Prevalence Rate of Cigarette Smoking among Congestive Heart Failure Patients at a Local Family Health Center. Ann Public Health Reports 3(1):30-34

als, healthcare providers, and supporting agencies frequently utilize evidence-based interventions (i.e. 3A's method) for smokers with $\mathrm{CHF}$ or comorbid conditions. Further research opportunities that will utilize secondary data from multiple study sites are highly recommended.

\section{Discussion}

Congestive heart failure is a common health problem in the U.S. As the prevalence rate of CHF continues to grow in the United States, this study provides current data from a small sample population retrieved from a local family clinic in San Bernardino County in Southern California. Although there was no random sampling that would provide empirical evidence for population generalization, there is an opportunity to answer the above questions and direct interventions that consider the clinic's clientele lifestyle practices. Approximately 600,000 people in the U.S. die each year because of $\mathrm{CHF}$ ( 1 out of every 9 deaths; [2]). For unknown reasons, research suggest that men $(71.8 \%)$ are more likely to die than women (39.1\%) from CHF [24]. In terms of age group distribution, those who are 55 years and older are more likely to die compared to those who are under 55 [24].

It is well documented in the literature that smoking is a major cause of cardiovascular diseases (CVD) and a risk factor that is responsible for 1 out of every 4 deaths [2]. Heart failure is not an exception, and that is the reason why the best thing all smokers are advised to do for their health is to kick off the habit. Some of the well-researched benefits that a smoker gains from quitting includes: Reduced risk for CVD which is said to happens immediately within 1 year, the risk of heart attack declines drops within 5 years, and the risk for stroke is lowered in comparison to that of a person who never smoked $[11,25,26]$. Additionally, when one quits smoking even for a few hours, their blood pressure and heart rate drops, which improves heart failure symptoms such as shortness of breath and general fatigue [27]. Hence, there is evidence that abstaining from smoking is be the best approach as there is no safe amount of smoking when it comes to patients with CHF. Research indicates that even a couple of cigarettes consumption a day are said to damage the heart, and that is why the one proven strategy for this population is to quit smoking $[11,27]$.

Our main findings are consistent with previous studies that have found smoking cessation to reduce morbidity and mortality in high-risk and general populations of smokers [26]. Smoking is associated with increased risk of CHF, but the risk decreases with increasing duration since smoking cessation has been implemented [25]. Despite knowing that smoking cessation reduces mortality and morbidity among patients with $\mathrm{CHF}$, some patients with $\mathrm{CHF}$ continue to smoke. Although quitting smoking will not cure $\mathrm{CHF}$, however, quitting will lessen chances of additional health problems. Possible interventions to help with smoking cessation among patients with CHF include a broader adoption of systematic cessation interventions within hospitals that advise patients about quitting, initiate cessation pharmacotherapies (mediations and counseling) and connect patients to follow-up support post-hospitalization [26].

\section{Study limitation}

This study focused on determining the prevalence rate of smoking among the adult population with $\mathrm{CHF}$ who were receiving medical care at the McKee FHC from January 1 to December 31, 2015. While McKee FHC is one of the 3 Ambulatory Care Centers of ARMC operating under one of the busiest teaching hospitals (with 456 beds) affiliated with the County of San Bernardino, no assumption has been made regarding the study's representation of a larger population. Additionally, the study exclusively utilized self-reported smoking status data from patient charts that did not include qualitative responses.

\section{Conclusion}

As the U.S. population continues to grow and many people tending to live longer due to advance practices of medicine, the health care impact of CHF will probably grow [24]. Hence more recent studies are encouraged to bring to light the prevalence rates and mortality rates of $\mathrm{CHF}$ in the U.S. not only among smokers but also among persons with other chronic conditions. Constant use of evidencebased smoking cessation intervention methods is highly recommended in all primary care centers, whereby providers frequently meet their high utilization patients such as those with heart failure. Healthcare providers in primary care settings have a vital role in encouraging their patients to quit smoking, particularly those suffering from chronic conditions such as: $\mathrm{CHF}$, cardiovascular diseases, respiratory diseases, diabetes, different type of cancers, and stroke [13]. Tobacco screening has and continues to be a very important part of daily assessment for the McKee FHC patients, whereby the $3 A^{\prime} s$ model for addressing nicotine dependence is employed as recommended by the Agency for Healthcare Research and Quality [12].

Overall, this study presented useful information pertaining to cigarette smoking among CHF patients from the McKee FHC and provided points of investigation for formulating evidencebased interventions. Although the prevalence rate of current smokers was significantly less than the prevalence rate of past smokers, it remains crucial for family physicians who frequently attend to patients with CHF to address cigarette smoking cessation behaviors. Questions posed by this study will hopefully provide directions for future investigations on effective evidence-based intervention strategies.

\section{Acknowledgement}

This study would like to acknowledge and thank the administration team at the McKee Family Health center, a division of Arrowhead Regional Medical Center for giving permission to study investigators to utilize their dataset and allow the research team to go through the Hospital IRB review process. Thank you.

\section{References}

1. Centers for Disease Control and Prevention (2016) Smoking \& tobacco use: Current cigarette smoking among adults in the united states. 
2. Centers for Disease Control and Prevention (2014) Division for heart disease and stroke prevention: Heart failure fact sheet.

3. Go AS, Mozaffarian D, Roger VL, et al. (2013) Heart disease and stroke statistics - 2013 update: A report from the American Heart Association. Circulation 127: e6-e245.

4. California Department of Public Health (2013) The burden of cardiovascular disease in California: Heart failure.

5. Centers for Disease Control and Prevention (2016) National Center for Health Statistics: Heart Disease.

6. National Institute of Health (2015) How does smoking affect the heart and blood vessel?

7. California Department of Public Health (2015) California tobacco facts and figures 2015: 25 years of tobacco control in California.

8. National Institute on Drug Abuse (2014) Tobacco/Nicotine and E-Cigs: Statistics and trends.

9. Okuyemi KS, Nollen NL, Ahluwalia JS (2006) Interventions to facilitate smoking cessation. Am Fam Physician 74: 262-271.

10. Office of Disease Prevention and Health Promotion, Healthy People 2020: Topics \& Objectives: Heart Disease and Stroke.

11. Centers for Disease Control and Prevention (2014) Smoking and cardiovascular disease: What you need to know about smoking and cardiovascular disease.

12. Agency for Healthcare Research and Quality (2012) Five major steps to interventions (The " $5 \mathrm{~A}^{\prime} \mathrm{s}$ ").

13. Centers for Disease Control and Prevention (2015) Health effects of cigarette smoking.

14. Ciccone Marco M, Aquilino A, Cortese F, et al. (2010) Feasibility and effectiveness of a disease and care management model in the primary health care system for patients with heart failure and diabetes (Project Leonardo). Vasc Health Risk Manag 6: 297305.
15. Centers for Disease Control and Prevention (2017) Extinguishing the tobacco epidemic in California: The problem.

16. Rigotti NA, Munafo MR, Stead LF (2008) Smoking cessation interventions for hospitalized smokers: A systematic review. Arch Intern Med 168: 1950-1960.

17. Rigotti NA, Clair C, Munafò MR, et al. (2012) Interventions for smoking cessation in hospitalized patients. Cochrane Database Syst Rev.

18. Stead LF, Koilpillai P, Fanshawe TR, et al. (2016) Combined pharmacotherapy and behavioral interventions for smoking cessation. Cochrane Database Syst Rev.

19. Prochaska JJ, Benowitz NL (2015) Smoking cessation and the cardiovascular patient. Curr Opin Cardiol 30: 506-511.

20. Prochaska J, Goldstein MG (1991) Process of smoking cessation. Implications for clinicians. Clin Chest Med 12: 727-735.

21. Gill GV, Morgan C, MacFarlane IA (2005) Awareness and use of smoking cessation treatments among diabetic patients. Diabet Med 22: 658-660.

22. Zimmerman G, Olsen CG, Bosworth MF (2000) A 'stages of change' approach to helping patients change behavior. Am Fam Physician 61: 1409-1416.

23. Martin LR, Williams SL, Haskard KB, et al. (2005) The challenge of patient adherence. Ther Clin Risk Manag 1: 189-199.

24. Schocken DD, Arrieta MI, Leaverton PE, et al. (1992) Prevalence and mortality rate of congestive heart failure in the United States. J Am Coll Cardiol 20: 301-306.

25. Aune D, Schlesinger S, Norat T, et al. (2018) Tobacco smoking and the risk of heart failure: A systematic review and metaanalysis of prospective studies. Eur J Prev Cardiol.

26. Mullen KA, Manuel DG, Hawken SJ, et al. (2017) Effectiveness of a hospital-initiated smoking cessation programme: 2-year health and healthcare outcomes. Tob Control 26: 293-299.

27. Health Library (HCA) How smoking affects your heart.

DOI: $10.36959 / 856 / 488$

Copyright: (C) 2019 Kapella-Mshigeni S, et al. This is an open-access article distributed under the terms of the Creative Commons Attribution License, which permits unrestricted use, distribution, and reproduction in any medium, provided the original author and source are credited. 